\title{
多色长余辉材料的发光性质及动态防伪应用
}

\author{
张聪, 李雨柔, 郡康, 林静, 王锴, 潘再法 \\ (浙江工业大学 化学工程学院, 杭州 310014)
}

摘 要: 发光防伪具有可视性强、设计简便的特点, 是众多防伪技术中常用的方法。传统防伪材料存在发光颜色单 一、防伪图案和颜色静态的缺点, 易于模仿, 亟需开发可实现动态、可靠防伪性能的发光材料。本工作采用水热法 制备了铬掺杂镓锗酸锌多色长余辉材料, 并对其余辉性能和动态防伪应用进行研究。实验结果表明: 通过改变镓锗 比, 可以调节蓝绿光和红光区的发射强度, 实现发光颜色的可调。该系列样品在波长为 254 和 $365 \mathrm{~nm}$ 的紫外光激 发下分别呈现白色和红色, 发光颜色具有多模态发光特征。此外该系列样品具有多色的余辉发光, 不同颜色的衰减 速率不同，可以实现余辉颜色随时间发生动态变化的效果。据此设计成的防伪图案，发光颜色在时间维度上具有动 态变化特性, 可显著提高防伪安全性, 表明所制备的铬掺杂镓锗酸锌多色长余辉材料在动态防伪领域有重要的应 用前景。

关 键 词: 长余辉; 多色发光; 动态防伪

中图分类号: O433 文献标志码: A

\section{Luminescence Property of the Multicolor Persistent Luminescence Materials for Dynamic Anti-counterfeiting Applications}

\author{
ZHANG Cong, LI Yurou, SHAO Kang, LIN Jing, WANG Kai, PAN Zaifa \\ (College of Chemical Engineering, Zhejiang University of Technology, Hangzhou 310014, China)
}

\begin{abstract}
Luminescent anti-counterfeiting has characteristics of visibility and convenience, which is a popular method in many anti-counterfeiting technologies. However, the anti-counterfeiting luminescent materials have the shortcomings of single emission color and static anti-counterfeiting pattern, leading to easy imitability. It is urgent to develop new luminescent materials that can achieve dynamic and more reliable anti-counterfeiting performance. In this research, the multicolor persistent luminescence material, chromium doped zinc gallogermanate, was prepared by hydrothermal method. Its persistent luminescence property and dynamic anti-counterfeiting application potential were investigated. Experimental results show that the emission intensity in blue-green and red light regions can be adjusted by changing the raw materials ratio of gallium to germanium. Under the excitation of 254 and $365 \mathrm{~nm}$ UV light, a series of samples are observed to be white and red, respectively, indicating multi-mode luminescence characteristics. Furthermore, the decay rate of blue, green and red components in white afterglow is different, so the afterglow color can change dynamically over time. Anti-counterfeiting patterns, designed based on
\end{abstract}

收稿日期：2021-01-25; 收到修改稿日期：2021-03-03; 网络出版日期：2021-04-05

基金项目：国家自然科学基金(10804099,21804119); 浙江省自然科学基金(LZ18B050002) National Natural Science Foundation of China (10804099, 21804119); Key Project of Natural Science Foundation of Zhejiang Province (LZ18B050002)

作者简介: 张 聪(1996-), 男, 硕士研究生. E-mail: 1765521429@qq.com ZHANG Cong(1996-), male, Master candidate. E-mail: 1765521429@qq.com

通信作者: 潘再法, 副教授. E-mail: panzaifa@zjut.edu.cn PAN Zaifa, associate professor. E-mail: panzaifa@zjut.edu.cn 
this multicolor afterglow feature, improve the security through dynamic change of afterglow color in the time dimension, demonstrating the potential application in dynamic anti-counterfeiting.

Key words: persistent luminescence; multicolor afterglow; dynamic anti-counterfeiting

随着信息时代的快速发展，信息安全也成为人 们普遍关注的问题。信息的防伪和加密技术是信息 安全的重要内容, 开发具有高可靠性的防伪技术已 经成为众多行业密切关注的问题 ${ }^{[1-2]}$ 。目前应用市场 上，除了传统刮开式的防伪技术，发光印花防伪技术 也日益增多。研究人员利用量子点 ${ }^{[3-4]}$, 有机染料 ${ }^{[-6-6]}$, 上转换发光材料 ${ }^{[7-8]}$ 和长余辉材料 ${ }^{[9-10]}$ 等制作发光印 花防伪标识。这些防伪标识在正常环境下不容易被 人眼识别, 只有在特定波段光的激发下才会显现, 与传统防伪技术相比, 其安全性有较大的提高。然 而这些防伪标识还存在一些缺点, 其防伪模式较为 单一, 大多采用单一波长激发荧光粉发光, 属于单 模态发光, 可模仿性较高。其次传统代码以静态形 式存储信息，不支持随时间变化的信息显示(例如， 在特定时间范围内消失的临时口令和文件), 其安 全可靠性有待进一步提高 ${ }^{[1-14]}$ 。因此, 需要开发一 种具有多模态发光以及发光动态变化特性的新型发 光材料, 用于提高防伪技术的安全性。

本工作通过水热法合成了一种具有多峰发射的 长余辉纳米材料铭掺杂镓锗酸锌 $(1-x) \mathrm{ZnGa}_{2} \mathrm{O}_{4}$ $x \mathrm{Zn}_{2} \mathrm{GeO}_{4}(\mathrm{ZGGO}: \mathrm{Cr})$, 并表征了其形貌结构和荧 光性能。在光致发光方面, 合成的多色 ZGGO: $\mathrm{Cr}$ 在不同激发波长下会展现出不同的颜色，属于多模 态发光, 以此实现多色防伪功能; 另外在余辉发光 方面, 各色光的余辉衰减速度不同致使发射颜色随 时间具有规律性的变化。基于以上重要特性, 将 ZGGO:Cr 制作成发光防伪图案, 通过发光颜色在时 间维度上的变化来实现动态防伪过程, 在信息安全 方面具有重要的应用前景。

\section{1 实验方法}

\section{1 实验试剂及仪器}

$\mathrm{Zn}\left(\mathrm{NO}_{3}\right)_{2} \cdot 6 \mathrm{H}_{2} \mathrm{O}(99.99 \%) 、 \mathrm{Ga}\left(\mathrm{NO}_{3}\right)_{3} \cdot x \mathrm{H}_{2} \mathrm{O}(99.9 \%)$ 、 $\mathrm{Cr}\left(\mathrm{NO}_{3}\right)_{3} \cdot 9 \mathrm{H}_{2} \mathrm{O}(99.95 \%)$ 均购自阿拉丁试剂有限公 司, $\mathrm{GeO}_{2}(99.99 \%)$ 购自南京细诺科技有限公司，氨 水 $(25 \%$ 28\%) 购自杭州龙山精细化工有限公司, $\mathrm{NaOH}(96 \%)$ 购自西龙科学股份有限公司、无水乙醇 ( $97 \%$ )购自安徽安特食品股份有限公司。
采用 $\chi^{\prime}$ pert PRO X 射线衍射仪(荷兰 PANlytical 公司)表征材料的物相组成, 采用 HT $7700120 \mathrm{kV}$ 透射电子显微镜(日本 Hitachi 公司)表征材料的结构 和形貌，采用 Fluoromax-4P 苂光光谱仪 (法国 HORIBA Jobin Yvon 公司)测试发光材料的荧光性能 (包括激发光谱、发射光谱和余辉衰减谱图), 采用佳 能 EOS R6 相机拍摄发光图案。

\section{2 实验步骤}

采用水热法制备铬掺杂长余辉纳米材料 $(1-x) \mathrm{ZnGa}_{2} \mathrm{O}_{4}$ $x \mathrm{Zn}_{2} \mathrm{GeO}_{4}(x=0.5,0.6,0.7,0.8,0.9)$, 铬离子掺杂浓度 为镓离子摩尔含量的 $0.5 \%$ 。具体过程如下: 分别配 制 $\mathrm{Zn}\left(\mathrm{NO}_{3}\right)_{2}(0.5 \mathrm{~mol} / \mathrm{L}) 、 \mathrm{Ga}\left(\mathrm{NO}_{3}\right)_{3}(0.5 \mathrm{~mol} / \mathrm{L})$ 溶液、 $\mathrm{Cr}\left(\mathrm{NO}_{3}\right)_{3}(0.01 \mathrm{~mol} / \mathrm{L})$ 溶液、 $\mathrm{Na}_{2} \mathrm{GeO}_{3}\left(0.5 \mathrm{~mol} / \mathrm{L}, \mathrm{GeO}_{2}\right.$ 与 $\mathrm{NaOH}$ 反应制备) 溶液备用。按配比量取一定体积 的 $\mathrm{Zn}\left(\mathrm{NO}_{3}\right)_{2} 、 \mathrm{Ga}\left(\mathrm{NO}_{3}\right)_{3} 、 \mathrm{Cr}\left(\mathrm{NO}_{3}\right)_{3}, \mathrm{Na}_{2} \mathrm{GeO}_{3}$ 母液加 入到 $20 \mathrm{~mL}$ 去离子水中, 随后在搅拌下缓慢加入氨水 以调节溶液的 $\mathrm{pH}$ 至 8 。摚拌 $1 \mathrm{~h}$ 后转移到 $30 \mathrm{~mL}$ 的反 应釜中，在烘箱中加热到 $220{ }^{\circ} \mathrm{C}$ 反应 $12 \mathrm{~h}$ 。反应完成 后, 待溶液冷却至室温, 通过离心处理(7000 r/min, $5 \mathrm{~min}$ )收集制备好的产物, 然后分别用去离子水、无 水乙醇洗涤产物(再分散后离心处理), 沉淀放入烘箱, $60{ }^{\circ} \mathrm{C}$ 干燥 $6 \mathrm{~h}$, 即得到 ZGGO : $\mathrm{Cr}$ 长余辉材料。

\section{2 结果与讨论}

\subsection{ZGGO : Cr 的结构形貌表征}

按通式 $(1-x) \mathrm{ZnGa}_{2} \mathrm{O}_{4}-x \mathrm{Zn}_{2} \mathrm{GeO}_{4}(x=0.5,0.6,0.7$, $0.8,0.9)$ 制备了不同化学计量比的长余辉材料, 探 究不同镓锗比对材料组成影响(如图 1 所示)。XRD 分析结果表明, 5 种比例的长余辉材料均由 $\mathrm{ZnGa}_{2} \mathrm{O}_{4}$ 和 $\mathrm{Zn}_{2} \mathrm{GeO}_{4}$ 两相组成。随着锗含量增加, $\mathrm{ZnGa}_{2} \mathrm{O}_{4}$ 相(PDF\#86-0413)的衍射峰强度减小, 逐渐向 $\mathrm{Zn}_{2} \mathrm{GeO}_{4}$ 相(PDF\#11-0687)过渡。因此本研究制备的 5 种多色 长余辉材料相当于不同比例的 $\mathrm{ZnGa}_{2} \mathrm{O}_{4}$ 和 $\mathrm{Zn}_{2} \mathrm{GeO}_{4}$ 两相组 成, 即 $(1-x) \mathrm{ZnGa}_{2} \mathrm{O}_{4}-x \mathrm{Zn}_{2} \mathrm{GeO}_{4}(x=0.5,0.6$, $0.7,0.8,0.9)$ 。实验样品与文献[15-16]报道的 ZGGO 不同之处在于，本实验中 $\mathrm{Zn}_{2} \mathrm{GeO}_{4}$ 晶型属于菱方晶 系(空间群为 $\mathrm{R} \overline{3})$, 而不是立方晶系 $(\mathrm{Fd} \overline{3} \mathrm{~m})$ 。菱形相的 $\mathrm{Zn}_{2} \mathrm{GeO}_{4}$ 在较高的压力和温度下会转变成立方相 ${ }^{[17]}$ 。 


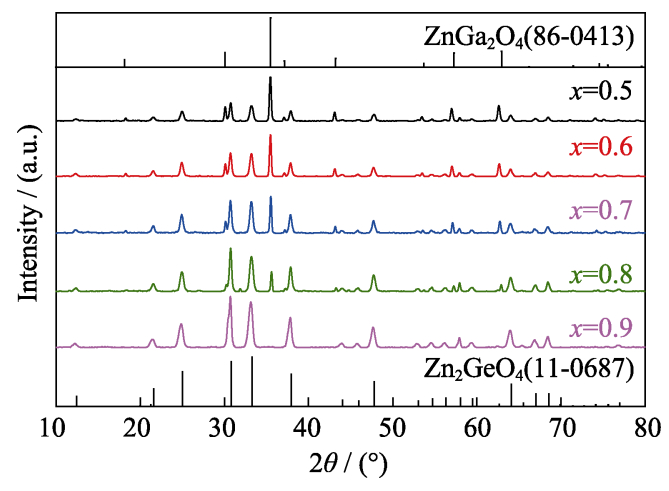

图 $1(1-x) \mathrm{ZnGa}_{2} \mathrm{O}_{4}-x \mathrm{Zn}_{2} \mathrm{GeO}_{4}(x=0.5,0.6,0.7,0.8,0.9)$ 的 $\mathrm{XRD}$ 图谱

Fig. 1 XRD patterns of $(1-x) \mathrm{ZnGa}_{2} \mathrm{O}_{4}-x \mathrm{Zn}_{2} \mathrm{GeO}_{4}(x=0.5,0.6$, $0.7,0.8,0.9)$

图 2 给出了不同基质组成 $\mathrm{ZGGO}: \mathrm{Cr}$ 的透射电 镜(TEM)照片, 从图中可知所有样品中包含了棒状 和不规则颗粒两种晶体。随着锗含量增加, 颗粒逐 渐减少, 棒状纳米晶的粒径逐渐从 $50 \mathrm{~nm}$ 增大到 $100 \mathrm{~nm}$ 左右，纳米粒子尺寸分布均匀。

\section{$2.2 \mathrm{ZGGO}: \mathrm{Cr}$ 的荧光性能表征}

进一步对 $(1-x) \mathrm{ZnGa}_{2} \mathrm{O}_{4}-x \mathrm{Zn}_{2} \mathrm{GeO}_{4}(x=0.5,0.6$, $0.7,0.8,0.9)$ 的苂光性能进行表征。图 3(a) 是 ZGGO : Cr 系列样品在 $273 \mathrm{~nm}$ 激发下的发射光谱, 不同镓锗比的样品均有两个发射峰。其中在蓝绿光 区是一个 400 600 $\mathrm{nm}$ 的宽峰, 对应于 ZGGO 基质 的本征发光 ${ }^{[18]}$ (详见 2.4)。ZGGO: $\mathrm{Cr}$ 系列样品在近 红外区存在一个 $696 \mathrm{~nm}$ 的窄带发射, 归因于 $\mathrm{Cr}^{3+}$ 离子的 ${ }^{2} \mathrm{E}\left({ }^{4} \mathrm{~F}\right) \rightarrow{ }^{4} \mathrm{~A}_{2}\left({ }^{4} \mathrm{~F}\right)$ 跃迁 ${ }^{[19-20]}$ 。如图 3(b) 所示, 随
着 $x$ 取值的增大, 蓝绿光发射逐渐增强, 而近红外 发光先增大后减小, $I_{696 \mathrm{~nm}} / I_{500 \mathrm{~nm}}$ 比值逐渐减小。可 见光区强度增加是由于 $\mathrm{Zn}_{2} \mathrm{GeO}_{4}$ 含量相对增加。从 XRD 谱图可知随着 $x$ 增大, $\mathrm{Zn}_{2} \mathrm{GaO}_{4}$ 相逐渐减少, $\mathrm{Zn}_{2} \mathrm{GaO}_{4}: \mathrm{Cr}^{3+}$ 含量减少导致近红外发射减弱。此外, ZGGO : Cr 的可见光发射与 $\mathrm{Cr}^{3+}$ 的激发带 ${ }^{4} \mathrm{~A}_{2} \rightarrow{ }^{4} \mathrm{~T}_{1}$ 跃迁(404 nm)和 ${ }^{4} \mathrm{~A}_{2} \rightarrow{ }^{4} \mathrm{~T}_{2}$ 跃迁(565 nm) 存在部分重 叠, 这有可能是 ZGGO: $\mathrm{Cr}(x=0.6)$ 的近红外发射增 强的原因。综上所述, 在相同的铬离子掺杂含量的 情况下, 可以通过改变镓锗比, 调节材料的多峰发 射，实现发光颜色的可调。

图 4(a) 是 $x=0.7$ 时样品的激发光谱, 当监测蓝光 和绿光发射峰时, 以 $254 \mathrm{~nm}$ 为中心的宽带激发峰来 自于 ZGGO 基质的带间吸收。当监测 $\mathrm{Cr}^{3+}$ 的近红外 发射时 ZGGO 有三个激发峰, 由于基质的带间跃迁 和 $\mathrm{O}-\mathrm{Cr}$ 电荷转移吸收带部分重叠, 在 $273 \mathrm{~nm}$ 处出 现了宽且强的激发峰; 404 和 $565 \mathrm{~nm}$ 分别对应于 $\mathrm{Cr}^{3+}$ 的 ${ }^{4} \mathrm{~A}_{2} \rightarrow{ }^{4} \mathrm{~T}_{1}$ 跃迁、 ${ }^{4} \mathrm{~A}_{2} \rightarrow{ }^{4} \mathrm{~T}_{2}$ 跃迁。蓝绿光只能 在激发波长小于 $300 \mathrm{~nm}$ 时激发, 而近红外光可以通 过紫外光或可见光激发。如图 4(b)所示, $x=0.7$ 的样 品在 $254 \mathrm{~nm}$ 激发下, 呈现多峰发射, 发光颜色为白 色。而在 $365 \mathrm{~nm}$ 激发下, 只有近红外发射, 样品为 红色。ZGGO : Cr 在不同的激发光下具有多模态发 射，可应用于信息的防伪加密，提升防伪的安全性。

\section{$2.3 \mathrm{ZGGO}: \mathrm{Cr}$ 的余辉性能表征}

对 ZGGO : $\mathrm{Cr}$ 的研究主要集中于 $\mathrm{Cr}^{3+}$ 的近红外 余辉性能, 例如 Pan 等 ${ }^{[15]}$ 认为 Ge 对 ZGGO 近红
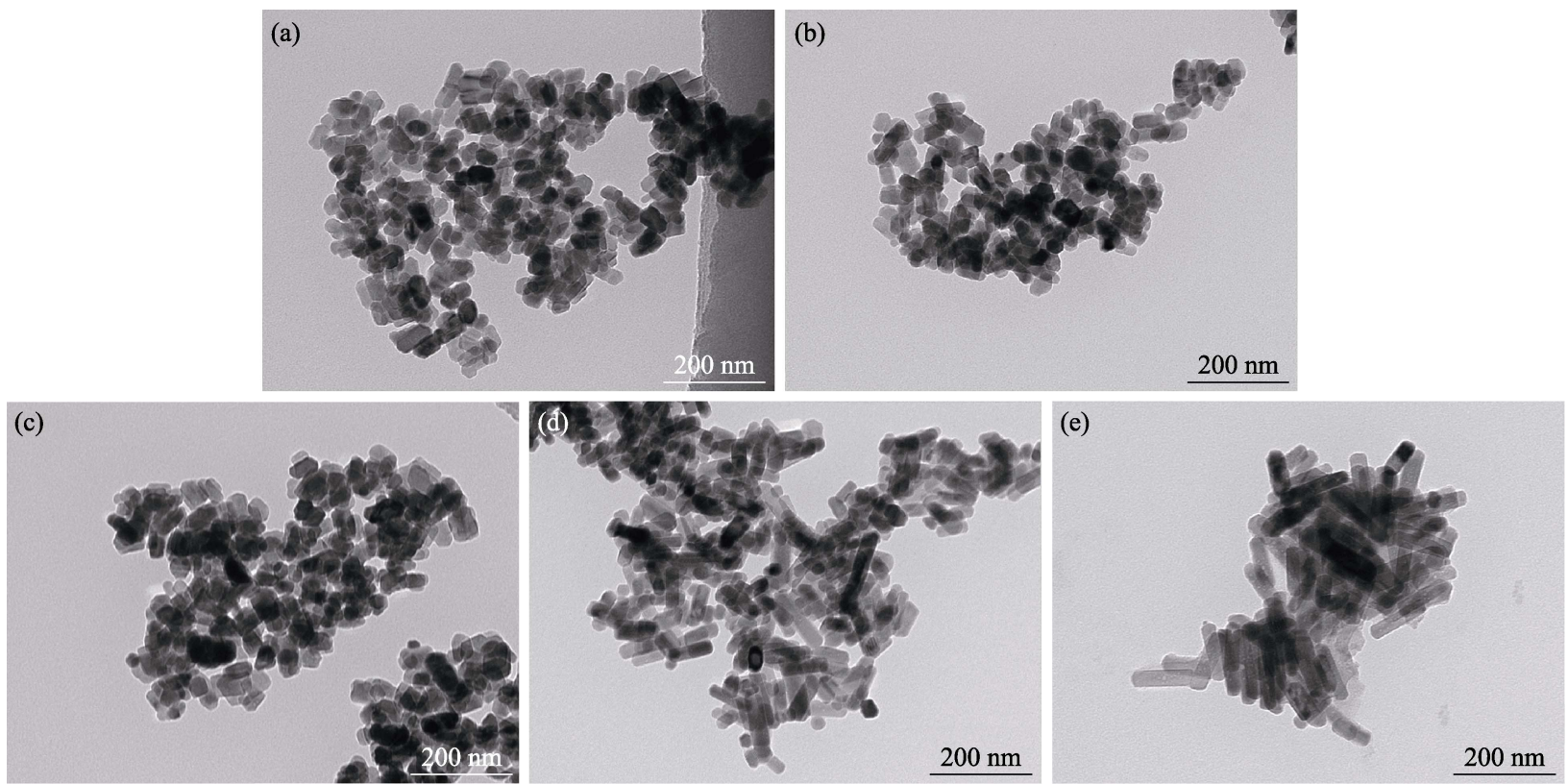

图 $2(1-x) \mathrm{ZnGa}_{2} \mathrm{O}_{4}-x \mathrm{Zn}_{2} \mathrm{GeO}_{4}$ 的 TEM 照片

Fig. 2 TEM images of $(1-x) \mathrm{ZnGa}_{2} \mathrm{O}_{4}-x \mathrm{Zn}_{2} \mathrm{GeO}_{4}$ (a) $x=0.5$; (b) $x=0.6$; (c) $x=0.7$; (d) $x=0.8$; (e) $x=0.9$ 

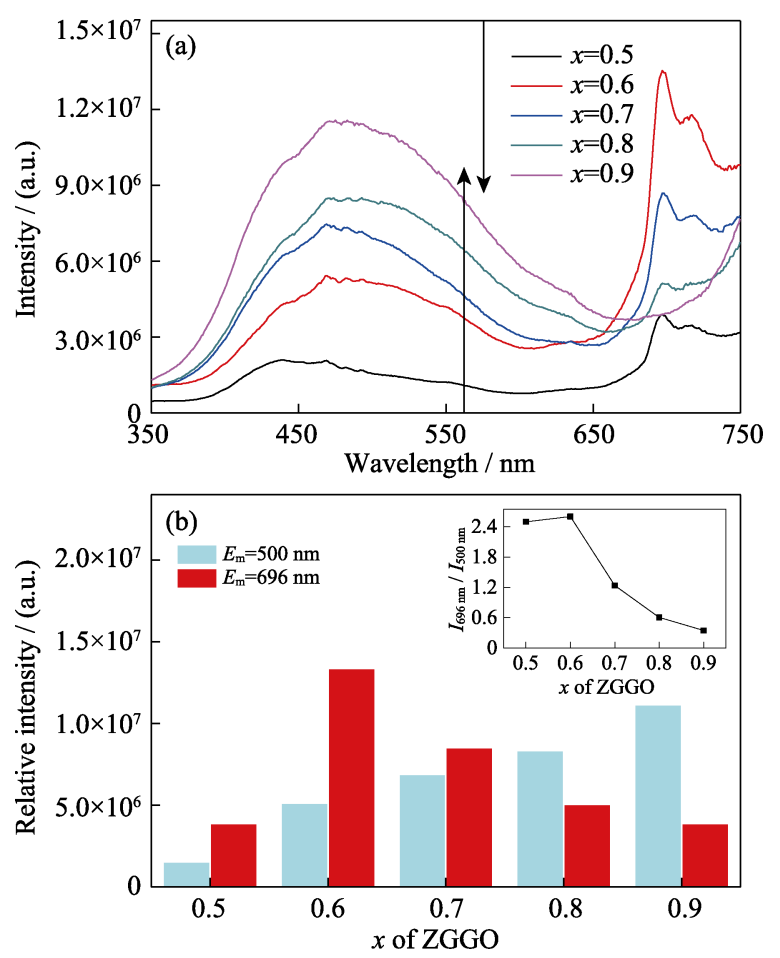

图 $3 \mathrm{ZGGO:Cr}$ 系列样品在 $273 \mathrm{~nm}$ 激发下的发射谱(a), ZGGO : $\mathrm{Cr}$ 系列样品在蓝绿光和近红外光区域的发光强度 的对比图(b), 插图是 $I_{696 \mathrm{~nm}} / I_{500 \mathrm{~nm}}$ 随 $x$ 变化的曲线

Fig. 3 Normalized emission spectra of ZGGO : Cr series samples under $273 \mathrm{~nm}$ excitation (a), and comparison of luminescence intensity of ZGGO : $\mathrm{Cr}$ series samples in blue-green and NIR region (b)

The inset in (b) shows the $I_{696 \mathrm{~nm}} / I_{500 \mathrm{~nm}}$ changing with $x$

外持续发光产生了重要影响, 带负电荷的 $\mathrm{V}_{\mathrm{Ge}}$ 和带 正电荷的 $\mathrm{V}_{\mathrm{O}}$ 可能位于 $\mathrm{Cr}^{3+}$ 离子附近, 形成 $\mathrm{V}_{\mathrm{Ge}}-\mathrm{Cr}^{3+}-\mathrm{V}_{\mathrm{O}}$ 缺陷团簇, 作为电子陷阱储存能量以获 得持续发光。本工作发现和探究了 $\mathrm{ZGGO}: \mathrm{Cr}$ 在可 见光区的余辉性能。图 5 是 ZGGO: Cr 系列样品在 蓝绿光和近红外光区的余辉衰减谱图。每次测试前 预先用氙灯激发 $5 \mathrm{~min}$, 然后在荧光光谱仪的 Kinetics 模式下分别监测蓝光 $(460 \mathrm{~nm}) 、$ 绿光 $(540 \mathrm{~nm})$ 和近红 外光(696 $\mathrm{nm}$ )处的发光强度, 获得了余辉强度随时 间变化的曲线。可以发现 ZGGO : Cr 系列样品在三
种波长下均有不同速率的衰减, 其中 $x=0.7$ 的样品 表现出较好的余辉性能。在激发光停止后的 $20 \mathrm{~s}$ 内, 三种波长下的余辉强度迅速下降，这是浅陷阱中的 电子快速释放导致的。 $20 \mathrm{~s}$ 后余辉强度逐渐衰减至 平缓, 此时衰减过程以深陷阱中电子的缓慢释放为 主，但深陷阱浓度较小，导致余辉较弱。

为提高材料的余辉性能, 将水热合成的 ZGGO : Cr 放入马弗炉中 $900{ }^{\circ} \mathrm{C}$ 煅烧 $1.5 \mathrm{~h}$ 。从图 6(a)中可以发 现, 经过高温处理后, ZGGO : $\mathrm{Cr}(x=0.7)$ 的发射峰明 显增强, 可见光区强度提升了 1.9 倍, 近红外光强度 提升了 2.5 倍。热处理对 ZGGO : Cr 材料余辉性
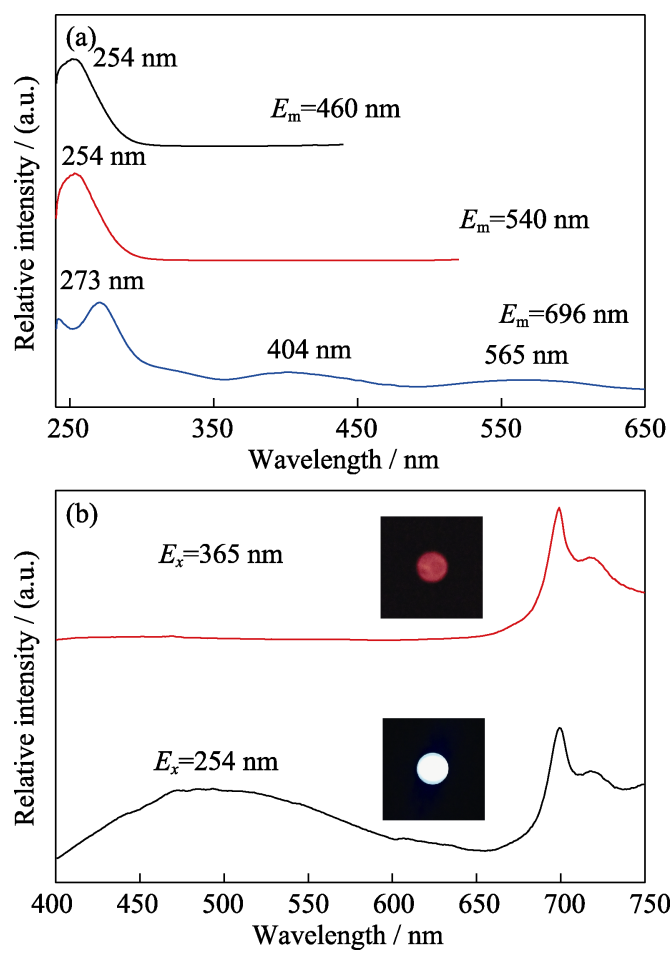

图 $4 x=0.7$ 时 ZGGO : $\mathrm{Cr}$ 的激发光谱 (a), $x=0.7$ 时 ZGGO : $\mathrm{Cr}$ 在 254 和 $365 \mathrm{~nm}$ 激发下的发射光谱和发光照片(b)

Fig. 4 Normalized excitation spectra of ZGGO : $\mathrm{Cr}(x=0.7)$ (a), and emission spectra of ZGGO : $\mathrm{Cr}(x=0.7)$ under $254 \mathrm{~nm}$ and $365 \mathrm{~nm}$ excitation (b)

Insets in (b) show the photographic images of the sample under 254 and $365 \mathrm{~nm}$ irradiation
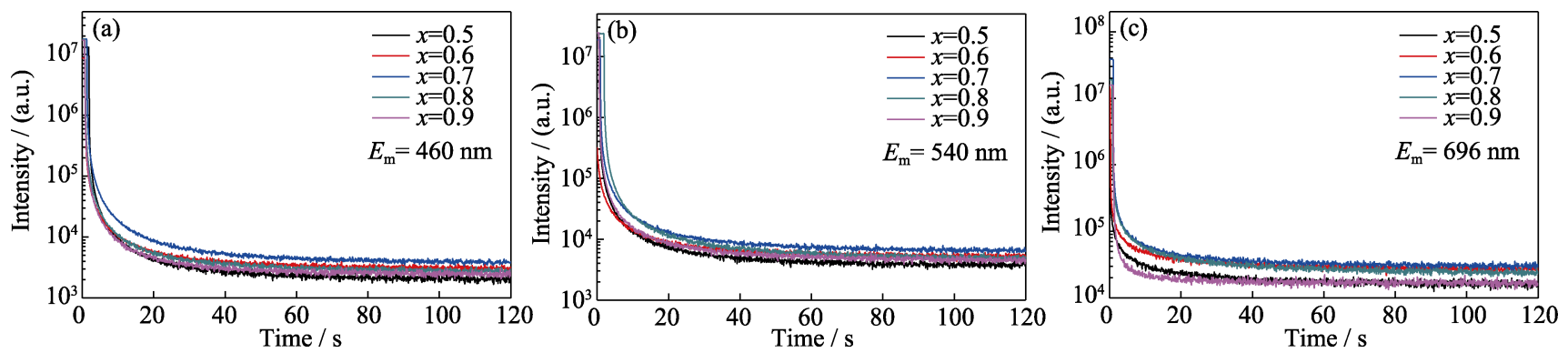

图 $5 \mathrm{ZGGO}: \mathrm{Cr}$ 系列样品的余辉衰减曲线(预先用氙灯充能 $5 \mathrm{~min}$ )

Fig. 5 Persistent luminescence decay curves of ZGGO : Cr with xenon lamp pre-charged for 5 min (a) $460 \mathrm{~nm}$; (b) $540 \mathrm{~nm}$; (c) $696 \mathrm{~nm}$ 

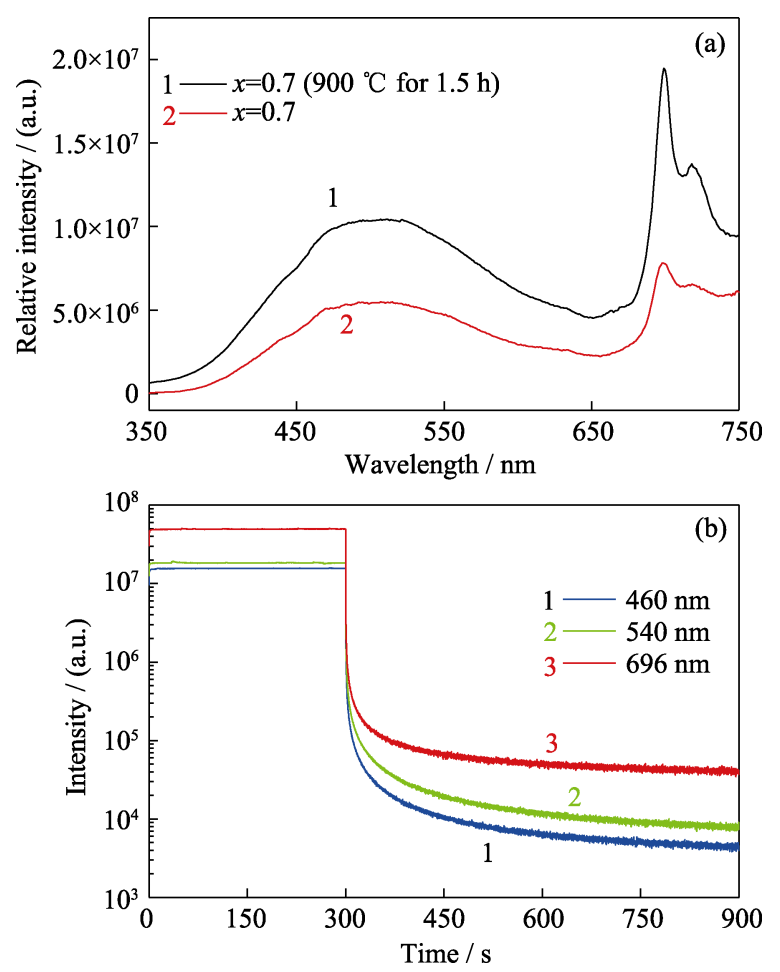

图 $6 \mathrm{ZGGO:} \mathrm{Cr}(x=0.7)$ 样品高温处理前后的发射谱图(a)和 $900{ }^{\circ} \mathrm{C}$ 㷽烧 $1.5 \mathrm{~h}$ 后的余辉衰减曲线(氙灯预先照射 $5 \mathrm{~min}$ )(b)

Fig. 6 Emission spectra of ZGGO : $\mathrm{Cr}(x=0.7)$ samples before and after high temperature treatment (a) and persistent luminescence decay curves of ZGGO : $\mathrm{Cr}$ after high temperature treatment with xenon lamp pre-charged for 5 min (b)

能影响的主要原因是高温有利于产生更多的热缺陷 (例如阳离子空位、阴离子空位和填隙型缺陷等), 从 而提升余辉性能 ${ }^{[21]}$ 。图 6(b) 为高温处理后 ZGGO : $\mathrm{Cr}(x=0.7)$ 在 $254 \mathrm{~nm}$ 充能 $5 \mathrm{~min}$ 的余辉衰减 曲线, 余辉时间和余辉强度明显增强。如图 6(b)所 示, 在余辉缓慢衰减的过程中, 绿、蓝两条衰减曲线 基本上是平行的, 而红光衰减速度较慢, 余辉强度 明显高于蓝绿光。可见光和近红外光的衰减速率不 同，致使 ZGGO : Cr 多峰持续性发光的颜色会发生 变化，这表明只能在某一时间窗观察到特定的颜 色。依图中趋势可知红光与蓝绿光发光强度差距会 越来越大，最终以红色为主导。

\subsection{ZGGO : Cr 的多色发光归属}

$\mathrm{ZGGO}: \mathrm{Cr}$ 样品包含 $\mathrm{ZnGa}_{2} \mathrm{O}_{4}$ 和 $\mathrm{Zn}_{2} \mathrm{GeO}_{4}$ 两相, 为了探究 ZGGO: $\mathrm{Cr}$ 多峰发射的来源, 本工作在相 同的水热条件下制备了纯的 $\mathrm{ZnGa}_{2} \mathrm{O}_{4}$ 和 $\mathrm{Zn}_{2} \mathrm{GeO}_{4}$ 基 质, 以及 $\mathrm{Cr}^{3+}$ 掺杂的样品 $\mathrm{ZnGa}_{2} \mathrm{O}_{4}: \mathrm{Cr}^{3+}$ 和 $\mathrm{Zn}_{2} \mathrm{GeO}_{4}: \mathrm{Cr}^{3+}$ 。其中前三个样品在 $254 \mathrm{~nm}$ 激发下, 发射峰分别在 440、530 和 $696 \mathrm{~nm}$ (如图 7 所示), $\mathrm{Zn}_{2} \mathrm{GeO}_{4}: \mathrm{Cr}^{3+}$ 样品则在蓝绿光区有宽带发光。

$\mathrm{ZnGa}_{2} \mathrm{O}_{4}$ 具有较宽的光学带隙 $(4.4 \sim 4.7 \mathrm{eV})$, 在紫外 光激发下能发出蓝光, 光谱范围为 $430 \sim 500 \mathrm{~nm}$ 左

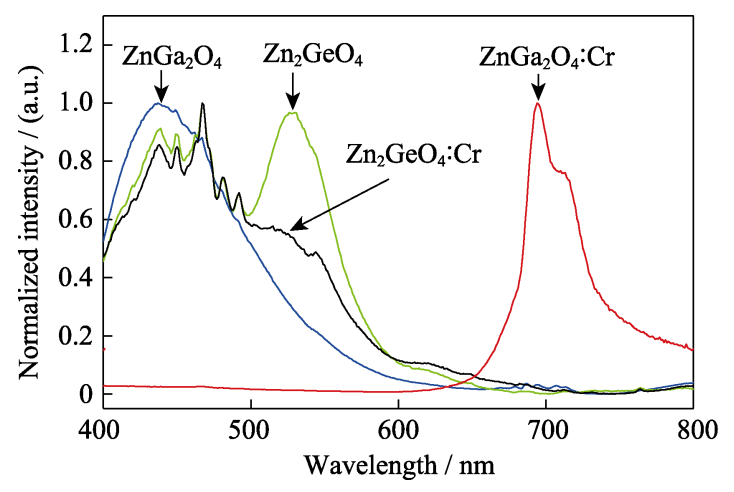

图 $7 \mathrm{ZnGa}_{2} \mathrm{O}_{4}, \mathrm{Zn}_{2} \mathrm{GeO}_{4}, \mathrm{Zn}_{2} \mathrm{GeO}_{4}: \mathrm{Cr}^{3+}$ 和 $\mathrm{ZnGa}_{2} \mathrm{O}_{4}: \mathrm{Cr}^{3+}$ 的 发射光谱 $\left(\lambda_{\mathrm{ex}}=254 \mathrm{~nm}\right)$

Fig. 7 Normalized emission spectra of $\mathrm{ZnGa}_{2} \mathrm{O}_{4}, \mathrm{Zn}_{2} \mathrm{GeO}_{4}$, $\mathrm{Zn}_{2} \mathrm{GeO}_{4}: \mathrm{Cr}^{3+}$, and $\mathrm{ZnGa}_{2} \mathrm{O}_{4}: \mathrm{Cr}^{3+}\left(\lambda_{\mathrm{ex}}=254 \mathrm{~nm}\right)$

右, 且具有纳秒级的苂光寿命。 $\mathrm{ZnGa}_{2} \mathrm{O}_{4}$ 的蓝光发 射来自于尖晶石中 $\mathrm{GaO}_{6}$ 基团自激活中心的跃迁 ${ }^{[22]}$ 。 此外有研究表明 $\mathrm{Zn}_{2} \mathrm{GeO}_{4}$ 是一种自激活的苂光粉, 内部存在大量本征缺陷, 在紫外光激发下, 电子陷 阱 $\left(V_{O}\right.$ 和 $\left.Z n_{i}\right)$ 与空穴陷阱 $\left(V_{\mathrm{Zn}}\right.$ 和 $\left.V_{\mathrm{Ge}}\right)$ 的复合导致 400 580 nm 的宽带发射 ${ }^{[23-25]}$ 。 $\mathrm{Zn}_{2} \mathrm{GeO}_{4}$ 本征缺陷 发光的波长和范围很大程度上取决于合成条件。例 如 $\mathrm{He}$ 等 ${ }^{[25]}$ 通过水热法制备了纯 $\mathrm{Zn}_{2} \mathrm{GeO}_{4}$, 在 $258 \mathrm{~nm}$ 激发下, 存在一个 $400 \sim 600 \mathrm{~nm}$ 宽带发射, 最大发 射波长在 $490 \mathrm{~nm}$ 。本实验结果与 $\mathrm{Chi}$ 等 ${ }^{[24]}$ 的研究 结果一致, 即在紫外光激发下纯的 $\mathrm{Zn}_{2} \mathrm{GeO}_{4}$ 在 430 和 $530 \mathrm{~nm}$ 左右存在两个发射峰(其中在 430 500 nm 范围的多个尖峰是来自于氙灯光源的杂散光干 扰)。430 nm 左右的发射是 $\mathrm{Zn}_{2} \mathrm{GeO}_{4}$ 中电子陷阱 $\left(\mathrm{V}_{\mathrm{O}}\right.$ 和 $\mathrm{Zn}_{\mathrm{i}}$ )与价带中空穴直接复合的结果, 而 $530 \mathrm{~nm}$ 左右的发射来自于电子陷阱 $\left(V_{O}\right.$ 和 $\left.Z_{i}\right)$ 与带隙中 空穴陷阱 $\left(V_{Z n}\right.$ 和 $\left.V_{G e}\right)$ 的复合。因此 ZGGO : $\mathrm{Cr}^{3+}$ 中的绿光发射来源于 $\mathrm{Zn}_{2} \mathrm{GeO}_{4}$ 的本征发光, 同时 部分蓝光发射可能也来自于 $\mathrm{Zn}_{2} \mathrm{GeO}_{4}$ 的发光。在 $\mathrm{ZnGa}_{2} \mathrm{O}_{4}: \mathrm{Cr}^{3+}$ 中, $\mathrm{Cr}^{3+}$ 与 $\mathrm{Ga}^{3+}$ 的半径和价态匹配, 通常 $\mathrm{Cr}^{3+}$ 占据的是 $\mathrm{Ga}^{3+}$ 的八面体格位, 会导致红 色或者近红外发射, 因此 $696 \mathrm{~nm}$ 的发光来自于 $\mathrm{ZnGa}_{2} \mathrm{O}_{4}: \mathrm{Cr}^{3+}$ 。而在 $\mathrm{Zn}_{2} \mathrm{GeO}_{4}: \mathrm{Cr}^{3+}$ 样品中由于 $\mathrm{Cr}^{3+}$ 占据 $\mathrm{Zn}^{2+}$ 或者 $\mathrm{Ge}^{4+}$ 的四面体格位, 并不会产 生近红外发光, 此外 $\mathrm{Cr}^{3+}$ 掺杂可能会导致 $\mathrm{Zn}_{2} \mathrm{GeO}_{4}$ 本征缺陷在 $530 \mathrm{~nm}$ 左右的发射减弱 ${ }^{[18]}$ 。 综上所述, $\mathrm{ZnGa}_{2} \mathrm{O}_{4}$ 发射峰在 $440 \mathrm{~nm}, \mathrm{Zn}_{2} \mathrm{GeO}_{4}$ 的 发光包含 430 和 $530 \mathrm{~nm}$ 两个发射峰, $\mathrm{ZnGa}_{2} \mathrm{O}_{4}$ : $\mathrm{Cr}^{3+}$ 的发射峰在 $696 \mathrm{~nm}, \mathrm{Zn}_{2} \mathrm{GeO}_{4}: \mathrm{Cr}^{3+}$ 也呈现了 430 和 $530 \mathrm{~nm}$ 两个发射峰, 但是 $530 \mathrm{~nm}$ 明显变 弱。ZGGO : Cr 中存在 $\mathrm{ZnGa}_{2} \mathrm{O}_{4}$ 和 $\mathrm{Zn}_{2} \mathrm{GeO}_{4}$ 两相, 所以 $x=0.7$ 样品的白色发光实际上包含上述多个 波长的复合发光。 
(a)
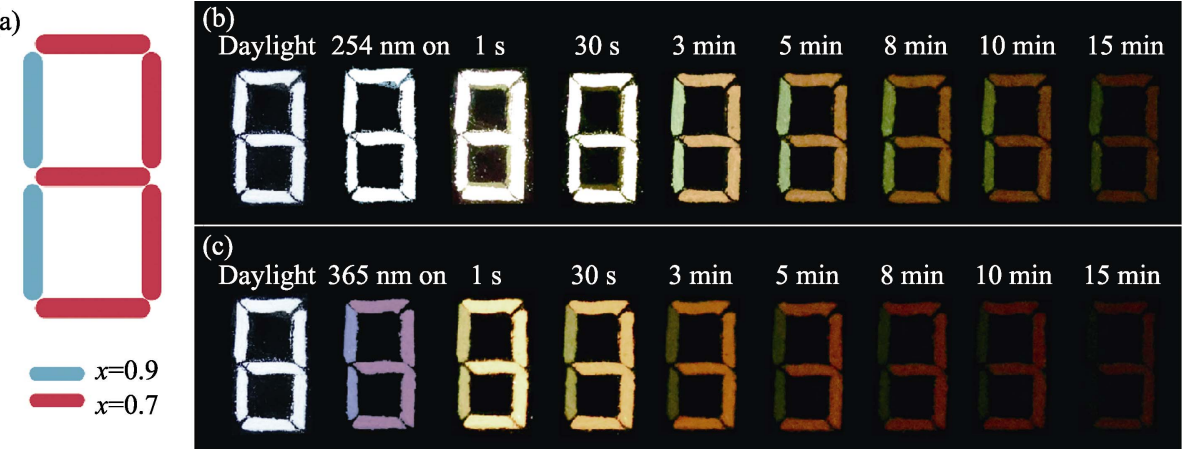

图 8 防伪图案制作原理(a), 发光图案在 $254 \mathrm{~nm}$ 紫外灯和不同衰减时间下的照片(b),

发光图案在 $365 \mathrm{~nm}$ 紫外灯和不同衰减时间下的照片(c)

Fig. 8 Manufacturing principle of anti-counterfeiting pattern (a), images of the luminescent pattern under $254 \mathrm{~nm}$ UV lamp and different decay time (b), and images of the luminescent pattern under $365 \mathrm{~nm}$ UV lamp and different decay time (c)

\section{3 动态防伪应用}

根据样品发光颜色在衰减过程中随时间不断改 变的特性，设计防伪图案。如图 8(a)所示，设计的图 案由两部分组成, 蓝色的“ 1 ”为 $x=0.9$ 的样品, 红色 的“3”为 $x=0.7$ 的样品。分别在 254 和 $365 \mathrm{~nm}$ 的紫 外灯下照射 $5 \mathrm{~min}$, 并用相机拍摄激发停止后的余 辉照片。

如图 8(b)所示, 在日光灯和 $254 \mathrm{~nm}$ 紫外灯下, $x=0.9$ 和 $x=0.7$ 的样品整体呈现不可分辨的白色, 图 案显示白色的“ 8 ”字样。当激发光停止后，随时间延 长, 整个图案逐渐由“8”变成颜色差异越来越大的 两部分“1”和“3”，这为信息安全提供了第一重保障。 此外图案的左半部分“8” 由白色逐渐转变为绿色, 而右半部分“3”由白色转变为红色。在整个衰减过程 中图案的颜色动态变化, 每个时间窗的颜色是唯一 的，动态防伪的方式难以复制，这是信息安全的第 二重保障。与 $254 \mathrm{~nm}$ 紫外灯的照射的情况不同, 在 $365 \mathrm{~nm}$ 紫外光照射下, 整个图案可以容易地分辨出 两部分“1”和“3”，而在 $15 \mathrm{~min}$ 的衰减过程中, “1”逐 渐消失，最后呈现红色“3”(如图 8(c))。因此，利用 254 和 $365 \mathrm{~nm}$ 紫外光对 ZGGO : Cr 中蓝绿光和近红 外光激发能力的不同, 实现了可靠性更高的动态防 伪, 为信息安全提供了第三重保障。以上设计的图 案可以用于设计防伪相关的产品, 通过发光颜色在 时间维度上的动态变化, 使得图案难以仿制, 为信 息安全提供多重保障, 可以很大程度提高防伪的安 全性。

\section{4 结论}

本研究采用水热法制备了 $(1-x) \mathrm{ZnGa}_{2} \mathrm{O}_{4}-x \mathrm{Zn}_{2} \mathrm{GeO}_{4}$
多色长余辉纳米材料。通过改变合成该材料时镓锗 的投料比，可以实现长余辉发光的颜色可调。该长 余辉材料在 254 和 $365 \mathrm{~nm}$ 光激发下分别呈现白色和 红色，其发光特性可应用于多模态发光防伪。组成 白光的三原色红、绿、蓝的余辉衰减速率不同, 致 使余辉颜色随时间发生动态变化，该特性可用于动 态防伪图案的设计，显著提高防伪加密的安全等级。

\section{参考文献:}

[1] ARPPE R, SØRENSEN T J. Physical unclonable functions generated through chemical methods for anti-counterfeiting. Nature Reviews Chemistry, 2017, 1(4): 0031.

[2] LIM K T P, LIU H, LIU Y, et al. Holographic colour prints for enhanced optical security by combined phase and amplitude control. Nature Communications, 2019, 10(1): 25-32.

[3] ZHU S, MENG Q, WANG L, et al. Highly photoluminescent carbon dots for multicolor patterning, sensors, and bioimaging. Angewandte Chemie International Edition, 2013, 52(14): 3953-3957.

[4] ZHOU W, ZHUANG J, LI W, et al. Towards efficient dual-emissive carbon dots through sulfur and nitrogen co-doped. Journal of Materials Chemistry C, 2017, 5(32): 8014-8021.

[5] WANG L, TAN W H. Multicolor FRET silica nanoparticles by single wavelength excitation. Nano Letters, 2006, 6(1): 84-88.

[6] KRUTZIK, P, NOLAN G. Fluorescent cell barcoding in flow cytometry allows high-throughput drug screening and signaling profiling. Nature Methods, 2006, 3: 361-368.

[7] ZHOU B, HUANG J, YAN L, et al. Probing energy migration through precise control of interfacial energy transfer in nanostructure. Advanced Materials, 2019, 31: 1806308.

[8] YAO W, TIAN Q, TIAN B, et al. Dual upconversion nanophotoswitch for security encoding. Science China Materials, 2019, 62(3): 368-378.

[9] ZHAO S, WANG Z, MA Z, et al. Achieving multimodal emission in $\mathrm{Zn}_{4} \mathrm{~B}_{6} \mathrm{O}_{13}: \mathrm{Tb}^{3+}, \mathrm{Yb}^{3+}$ for information encryption and anticounterfeiting. Inorganic Chemistry, 2020, 59(21): 15681-15689.

[10] WANG J, MA Q, ZHENG W, et al. One-dimensional luminous nanorods featuring tunable persistent luminescence for autofluorescence-free biosensing. ACS Nano, 2017, 11(8): 8185-8191.

[11] GU L, SHI H, GU M, et al. Dynamic ultralong organic phosphorescence by photoactivation. Angewandte Chemie International 
Edition, 2018, 57(28): 8425-8431.

[12] KALININ Y V, PANDEY S, HONG J, et al. A chemical display: generating animations by controlled diffusion from porous voxels. Advanced Functional Materials, 2015, 25(26): 3998-4004.

[13] ZHANG Y, HUANG R, LI H, et al. Triple-mode emissions with invisible near-infrared after-glow from $\mathrm{Cr}^{3+}$-doped zinc aluminum germanium nanoparticles for advanced anti-counterfeiting applications. Small, 2020, 16(35): 2003121.

[14] GANGWAR A K, KANIKA K, KEDAWAT G, et al. Single excitable dual emissive novel luminescent pigment to generate advanced security features for anti-counterfeiting applications. Journal of Materials Chemistry C, 2019, 7(44): 13867-13877.

[15] PAN Z, LU Y, LIU F. Sunlight-activated long-persistent luminescence in the near-infrared from $\mathrm{Cr}^{3+}$-doped zinc gallogermanates. Nature Materials, 2012, 11(1): 58-63.

[16] LI J Y, YAN X P. Synthesis of functionalized triple-doped zinc gallogermanate nanoparticles with superlong near-infrared persistent luminescence for long-term orally administrated bioimaging. Nanoscale, 2016, 8: 14965-14970.

[17] LI L, PAN F, TANNER P A, et al. Tunable dual visible and nearinfrared persistent luminescence in doped zinc gallogermanate nanoparticles for simultaneous photosensitization and bioimaging. ACS Applied Nano Materials, 2020, 3(2): 1961-1971.

[18] BAI Q, LI P, WANG Z, et al. Inducing tunable host luminescence in $\mathrm{Zn}_{2} \mathrm{GeO}_{4}$ tetrahedral materials via doping $\mathrm{Cr}^{3+}$. Spectrochimica Acta Part A: Molecular and Biomolecular Spectroscopy, 2018, 199:
$179-188$.

[19] ZHOU Z H, ZHENG W, KONG J T, et al. Rechargeable and LEDactivated $\mathrm{ZnGa}_{2} \mathrm{O}_{4}: \mathrm{Cr}^{3+}$ near-infrared persistent luminescence nanoprobes for background-free biodetection. Nanoscale, 2017, 9: 6846-6853.

[20] WANG K, YAN L P, SHAO K, et al. Near-infrared afterglow enhancement and trap distribution analysis of silicon-chromium co-doped persistent luminescence materials $\mathrm{Zn}_{1+x} \mathrm{Ga}_{2-2 x} \mathrm{Si}_{x} \mathrm{O}_{4}: \mathrm{Cr}^{3+}$. Journal of Inorganic Materials, 2019, 34(9): 983-990.

[21] LI Y, GECEVICIUS M, QIU J R. Long persistent phosphors-from fundamentals to applications. Chemical Society Reviews, 2016, 45(8): 2090-2136.

[22] ZHANG Y, WU Z, GENG D, et al. Full color emission in $\mathrm{ZnGa}_{2} \mathrm{O}_{4}$ : simultaneous control of the spherical morphology, luminescent, and electric properties via hydrothermal approach. Advanced Functional Materials, 2014, 24(42): 6581-6593.

[23] LIU Z, JING X, WANG L. Luminescence of native defects in $\mathrm{Zn}_{2} \mathrm{GeO}_{4}$. Journal of the Electrochemical Society, 2007, 154(6): $\mathrm{H} 500-\mathrm{H} 506$.

[24] CHI F F, WEI X T, JIANG B, et al. Luminescence properties and the thermal quenching mechanism of $\mathrm{Mn}^{2+}$ doped $\mathrm{Zn}_{2} \mathrm{GeO}_{4}$ long persistent phosphors. Dalton Transactions, 2018, 47(4): 1303-1311.

[25] HE H, ZHANG Y, PAN Q, et al. Controllable synthesis of $\mathrm{Zn}_{2} \mathrm{GeO}_{4}: \mathrm{Eu}$ nanocrystals with multi-color emission for white light-emitting diodes. Journal of Materials Chemistry C, 2015, 3(21): 5419-5429. 